Technical Note

\title{
Laser Doppler imaging for intraoperative human brain mapping
}

\author{
A. Raabe ${ }^{a}$, D. Van De Ville ${ }^{b}$, M. Leutenegger ${ }^{c}, A$. Szelényi ${ }^{a}$, E. Hattingen ${ }^{\text {d }}$, R. Gerlach ${ }^{\text {a }}$, V. Seifert ${ }^{\text {a }}$, \\ C. Hauger ${ }^{\mathrm{e}}$, A. Lopez $^{\mathrm{c}}$, R. Leitgeb ${ }^{\mathrm{c}}$, M. Unser ${ }^{\text {b }}$, E.J. Martin-Williams ${ }^{\mathrm{c}}$, T. Lasser $^{\mathrm{c}, *}$ \\ a Department of Neurosurgery, Johann Wolfgang Goethe University, Frankfurt am Main, Germany \\ ${ }^{\mathrm{b}}$ Biomedical Imaging Group, Ecole Polytechnique Fédérale de Lausanne, Lausanne, Switzerland \\ c Laboratory of Biomedical Optics, Ecole Polytechnique Fédérale de Lausanne, Lausanne, Switzerland \\ d Institute of Neuroradiology, Johann Wolfgang Goethe University, Frankfurt am Main, Germany \\ e Carl Zeiss Meditec AG, Oberkochen, Germany
}

\section{A R T I C L E I N F O}

\section{Article history:}

Received 16 September 2008

Revised 27 October 2008

Accepted 28 October 2008

Available online 12 November 2008

\begin{abstract}
A B S T R A C T
The identification and accurate location of centers of brain activity are vital both in neuro-surgery and brain research. This study aimed to provide a non-invasive, non-contact, accurate, rapid and user-friendly means of producing functional images intraoperatively. To this end a full field Laser Doppler imager was developed and integrated within the surgical microscope and perfusion images of the cortical surface were acquired during awake surgery whilst the patient performed a predetermined task. The regions of brain activity showed a clear signal (10-20\% with respect to the baseline) related to the stimulation protocol which lead to intraoperative functional brain maps of strong statistical significance and which correlate well with the preoperative fMRI and intraoperative cortical electro-stimulation. These initial results achieved with a prototype device and wavelet based regressor analysis (the hemodynamic response function being derived from MRI applications) demonstrate the feasibility of LDI as an appropriate technique for intraoperative functional brain imaging.
\end{abstract}

(C) 2008 Elsevier Inc. All rights reserved.

\section{Introduction}

Over recent years there has been a flourish of research furthering our understanding of brain function, connectivity, hemodynamics, chemistry, mapping etc; this progress has been made hand in hand with the development of a plethora of techniques to probe or observe the brain in action. These techniques range from fMRI, PET and Laser speckle imaging to optical intrinsic signal imaging and near-infrared spectroscopy amongst others (Hillman, 2007; Obrig and Villringer, 2003; Toga and Mazziotta, 2002; Vennemann et al., 2007). Each technique adds its portion of information to the puzzle but also brings with it its own specific technical limitations.

The surgical removal of a lesion adjacent to motor, sensory, language or visuospatial areas requires their identification in order that these functionally-important regions of the brain may be preserved. Neurosurgery has gained considerably in precision over recent years thanks to the availability of methods for visualizing the brain and its functioning, such as the use of fMRI (Toga and Mazziotta, 2002; Logothetis and Pfeuffer, 2004) for brain mapping, the integration of fMRI into neuronavigation tools for use during surgery together with cortical electrostimulation (Berman et al., 2004). FMRI results are complicated by 'brain shift' necessitating the recalculation of naviga-

\footnotetext{
* Corresponding author. Laboratoire d'optique biomédicale, EPFL STI LOB, Bâtiment BM, Station 17, CH-1015 Lausanne, Switzerland. Fax: +41 216937820.

E-mail address: theo.lasser@epfl.ch (T. Lasser).
}

tion data during theatre, and by the large vessel effect extending the signal towards the draining vessels. Electro-cortical stimulation is the surgical gold standard and directly reveals interference with brain function, but it is a laborious and time consuming technique yielding occasional false positives and exposing the patient to the risk of seizure. Thus there remains need for a rapid, high resolution, noninvasive method applicable in theatre allowing the visualization of areas of cortical activation across the exposed brain, i.e. a wide field of view, as well as enabling many different and complex functional maps to be generated intraoperatively.

Visualizing blood perfusion related phenomena is an indirect but standard method for observing brain function, the link between neuronal activity, metabolic demand and blood flow having been recognized for many years. The spatiotemporal details however, are still subject to active research as are the exact cellular mechanisms involved. Furthermore the need to be able to test awake subjects has become more obvious as the effects of anesthetics on regulation mechanisms have become apparent (Iadecola and Nedergaard, 2007). These perfusion related functional variations can be visualized using optics-based techniques. The use of optical intrinsic signal imaging (OIS) for intraoperative brain mapping was pioneered by (Haglund et al., 1992) producing functional maps of high temporal resolution but requiring a glass plate to be in contact with the cortex (to minimize movement related artefacts) and several task repetitions to achieve a reliable signal to noise ratio. Intraoperative OIS has been developed further to incorporate image alignment and analysis, 
mostly eliminating the need for a glass plate (Pouratian et al., 2002a, 2003) and has provided many advances in high resolution brain mapping as well as understanding of local hemodynamics. Methods based on Laser Doppler provide another possibility, our work has derived from a CMOS-based Laser Doppler Imager (LDI) which has been designed and constructed to fit on a standard surgical microscope. The perfusion images we present here were taken during open brain surgery with an awake patient performing specific tasks. These functional images have been post-processed using a statistical framework that performs a least-squares fit of the various regressors. The temporal general linear model includes the modeled stimulusinduced response and also periodic components to capture breathing, pulse and vasomotion. The stimulus-related signal has then been extracted and hypothesis testing performed to evaluate the evidence of its presence.

\section{Materials and methods}

The observations were carried out with the approval of the ethics committee of the University Clinics of Frankfurt am Main and with the patient's written informed consent. Prior to entering theatre, the equipment underwent a full risk analysis, CE homologation and was approved by an independent authorized medical homologation body for neurosurgery. The LDI system was designed to be of Laser Class 1.

The LDI (Serov and Lasser, 2005) was integrated beneath a surgical microscope (Zeiss Pico). The imaging system uses a fast CMOS camera as detector and as such is a full field imager designed to image the area of interest (currently $4 \times 3.5 \mathrm{~cm}$ at $30 \mathrm{~cm}$ distance and $140 \times 120$ pixels). This area is imaged with white light, producing a conventional optical image; as well as with a monomode Laser emitting at $808 \mathrm{~nm}$ which generates the LDI image at $1.48 \mathrm{~Hz}$ (the perfusion image being derived from the negative of the first order moment due to the camera speed). The wavelength chosen allows the imaging of total blood flow; the absorption of oxy- and deoxy-hemoglobin being essentially equal at this wavelength (Wray et al., 1988).

A male patient (44 years) with an anaplastic astrocytoma (WHO ${ }^{\circ}$ III) close to the dominant (left sided) dorsal inferior frontal gyrus was investigated. Before surgery a functional MRI was performed so as to identify the hand, foot and tongue motor centers, and integrated in the neuronavigation 3D data set. The functional magnetic resonance imaging was performed with a 1.5 Tesla Siemens Magnetom Vision scanner (Siemens AG, Erlangen, Germany) using the echo-planar T2* imaging technique (EPI; 15 slices, slice thickness $6 \mathrm{~mm}$, slice distance 0,6 mm, TE $66 \mathrm{~ms}$, TR $4 \mathrm{sec}$, matrix $128 \times 128$, FOV $230 \mathrm{~mm}, 120$ measurements, and 3 dummy measurements). The paradigm used consisted of 8 periods of alternating tongue movements along the teeth as well as movements of the hand (finger tapping) and foot (toe flexing) contra-lateral to the affected side. No resting condition was implemented. A T1 weighted 3D MP-RAGE data set was acquired as anatomical reference scan, the voxel size being $1 \times 1 \times 1 \mathrm{~mm}$. FMRI data analysis was performed using the BrainVoyager Software (Brain Innovation, Maastricht, NL). 2D- and 3D-functional maps were calculated based on a cross correlation analysis between the measured and presumed signal time course for each voxel after motion correction. The MRI Mercator view was generated including the activation map for the task. A laser surface scanning device (z-touch ${ }^{\circledR}$, BrainLAB, Heimstetten, Germany) was used for patient registration and the BrainLAB VectorVision for neuronavigation. The craniotomy was performed with the patient's head immobilized in a Mayfield headrest system (OMI, Inc., Cincinnati, OH, USA) ensuring a rigid mechanical connection between the head holder and the reference star. Extreme positioning was avoided to minimize brain shift and venous congestion. A registration accuracy check was performed on anatomical landmarks with accuracy better than $1.8 \mathrm{~mm}$.

The craniotomy was performed under local anesthesia so that the patient was fully awake during surgery. Once open, the whole exposed cortex was mapped for motor and language function at $5 \mathrm{~mm}$ intervals (determined by the probe dimensions) by standard cortical electrostimulation, this was achieved with rectangular pulses of frequency $50-60 \mathrm{~Hz}$ and 1-4 mA, the current being increased by $0.5 \mathrm{~mA}$ at each trial. The stimulation duration was of $1 \mathrm{~s}$ for sensorimotor and $4 \mathrm{~s}$ for cognitive and language functions. The areas proven to be active after at least three nonconsecutive trials were then labeled.

After identification of the motor hand map, the patient was asked to perform a finger tapping task to generate the activation for the LDI imaging. Fig. 1 provides an overview of the sequence of steps necessary for obtaining a functional image. Before starting the finger tapping we obtained an initial rest baseline with the patients eyes closed and quiet in the operating theatre for $\sim 2-3 \mathrm{~min}$. Once this had been achieved, each epoch of finger-tapping was initiated and terminated by a visual cue. The rest periods between epochs were of varying lengths to eliminate any spurious low frequency interference. Breathing and heart rates were also noted for inclusion in the regression analysis.

The time-series of perfusion images was then analyzed using confirmatory statistical analysis that looks for evidence of the stimulus-related signal. The flowchart of the complete data postprocessing pipeline is shown in Fig. 2. The time-series of perfusion
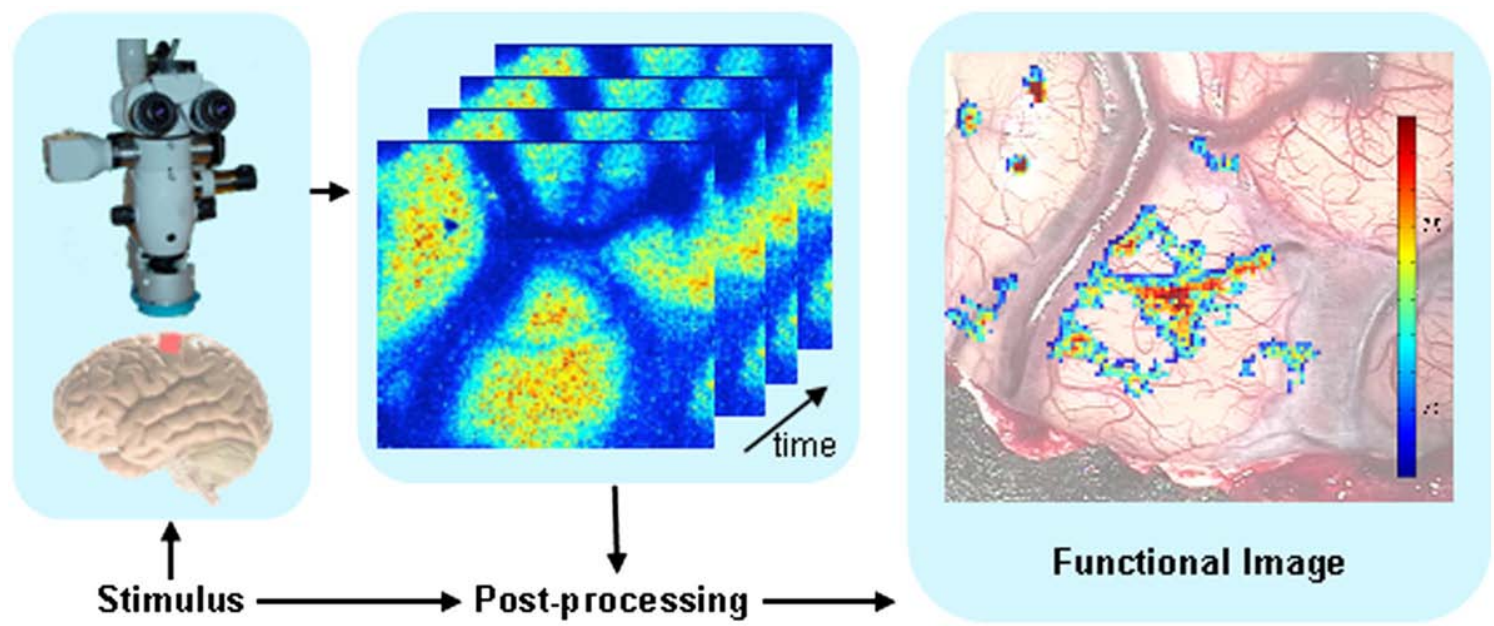

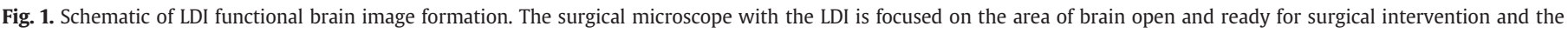

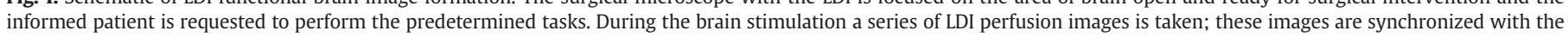

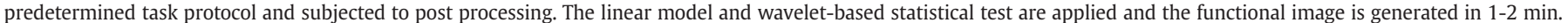



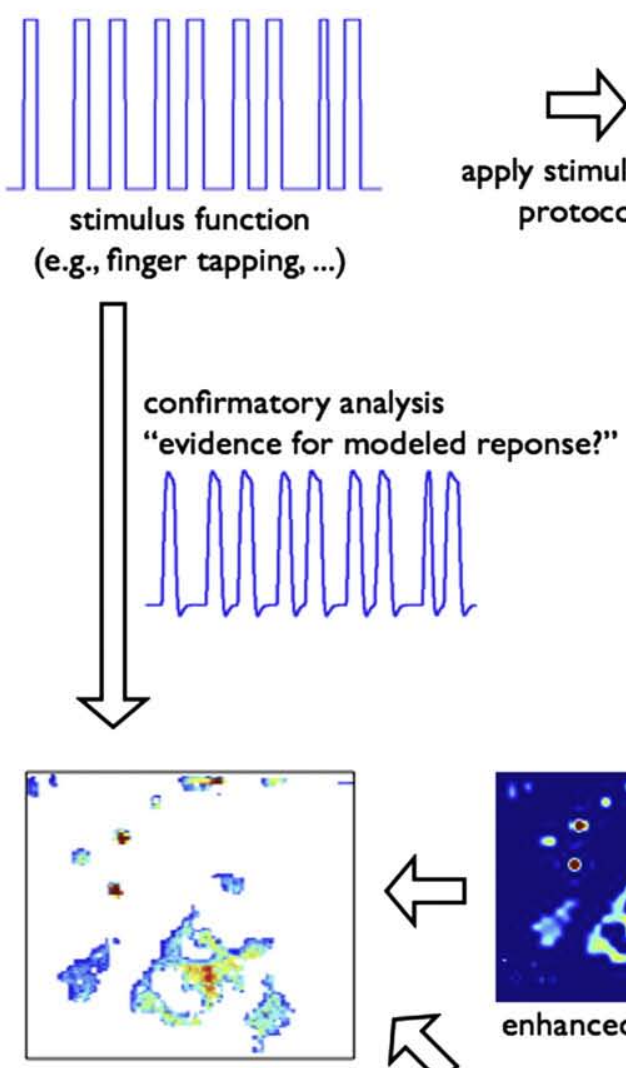

statistical parametric map

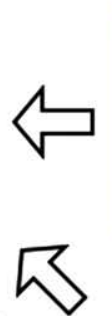

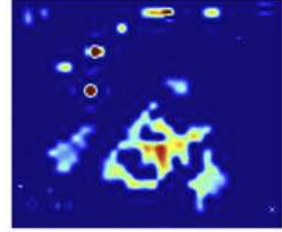

enhanced contrast

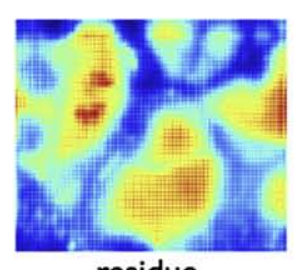

residue
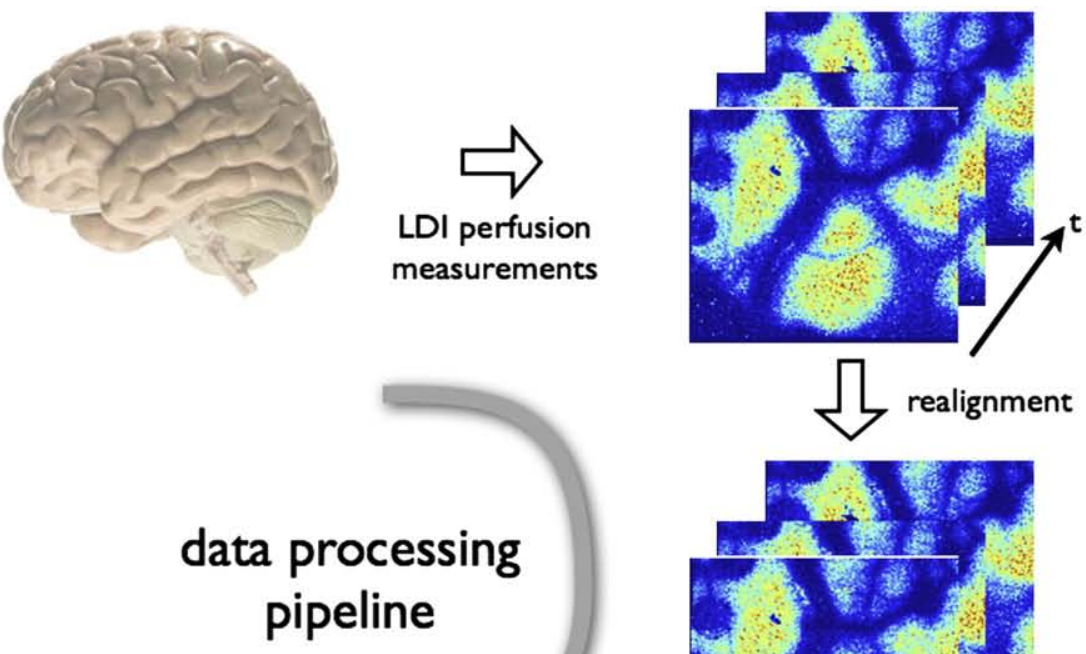
pipeline
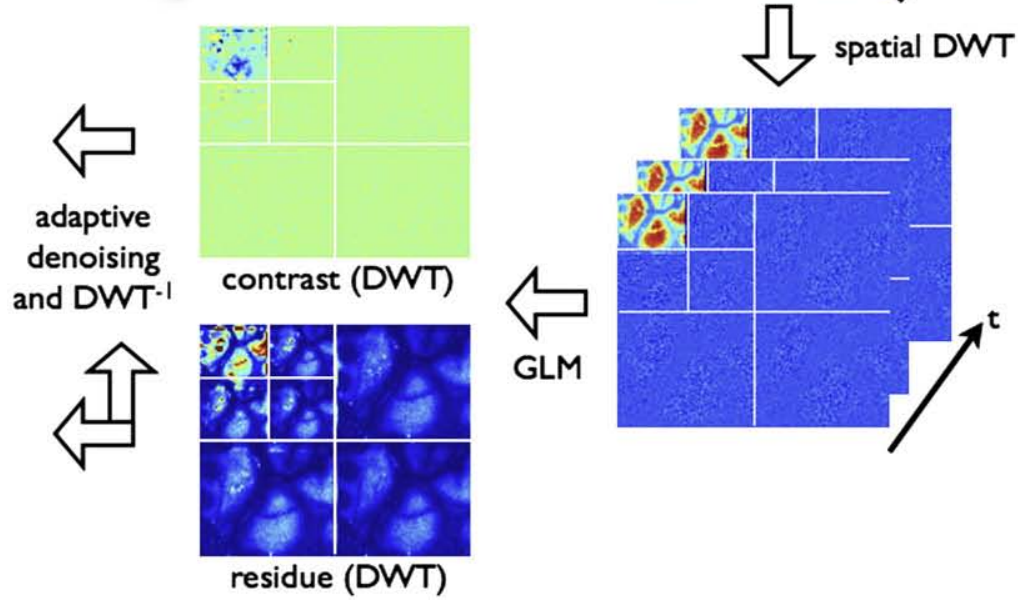

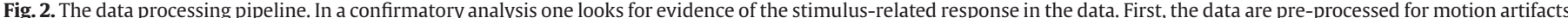

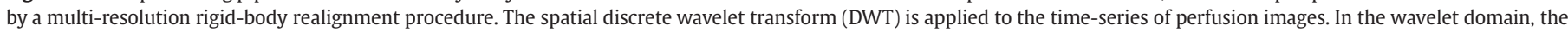

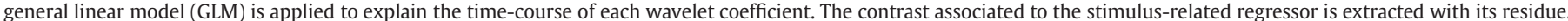

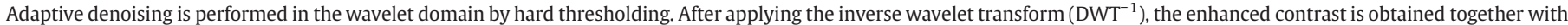
a spatial estimate of the residue. Finally, statistical hypothesis testing results in the statistical parametric map for the stimulus condition.

images was pre-processed to compensate for small motion artifacts using a robust multi-resolution rigid-body registration algorithm (Thévenaz et al., 1998). The time-course of each pixel is then explained by a general linear model comprising various regressors (Frackowiak, 2004; Bullmore et al., 1996). Within this model, the activity-related regressor is modeled as the stimulus function convolved with a hemodynamic response function for the local cerebral blood flow taken from the fMRI literature and using a time to peak of $3 \mathrm{~s}$ and a post stimulus undershoot (Buxton and Frank, 1997; Friston et al., 2000). Additional regressors include; the baseline, linear drift, low-pass DCT basis (up to $0.01 \mathrm{~Hz}$ ) and periodic components at $0.1 \mathrm{~Hz}$ (vasomotion) and $0.36 \mathrm{~Hz}$ (respiration). The framework makes use of the spatial wavelet transform (two dimensional Battle-Lemarié wavelets) to efficiently exploit the spatial correlation present in the data (Van De
Ville et al., 2004). This allows the combination of denoising in the wavelet domain with statistical testing in the spatial domain.

\section{Results and discussion}

Fig. 3(a) shows the statistical map for the task-related regressor for a confidence level of $0.01 \%$ (corrected for multiple comparisons) superposed on the conventional optical image. In Fig. 3(b), the average time-course of the most-activated region is extracted after removal of the non-stimulus related regressors. The activation related signal change is of a magnitude of $10-20 \%$ with respect to the baseline (corresponding to the t-values shown in Figs. 1 and 3(a) depicted by the color scale). The stimulus (blue) and blood-flow regressors (black) are also shown. Finally, in Fig. 3(c), we show the

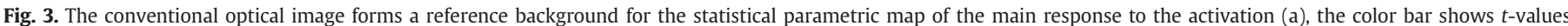

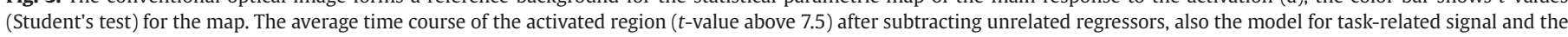

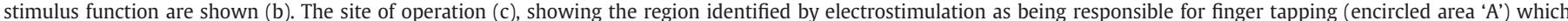

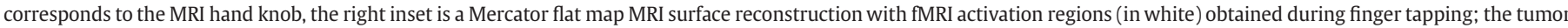

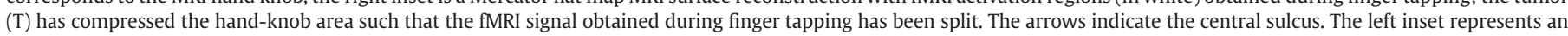
enlarged image corresponding to Fig. 2(a). 
a

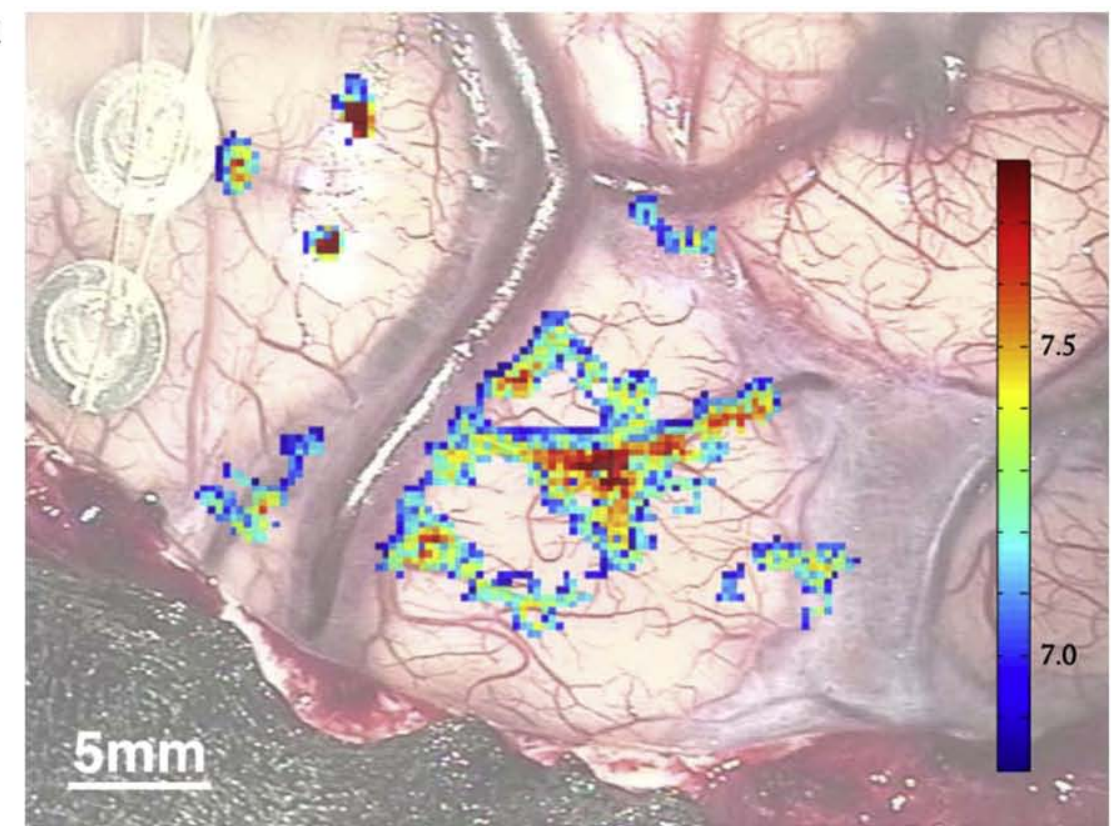

b

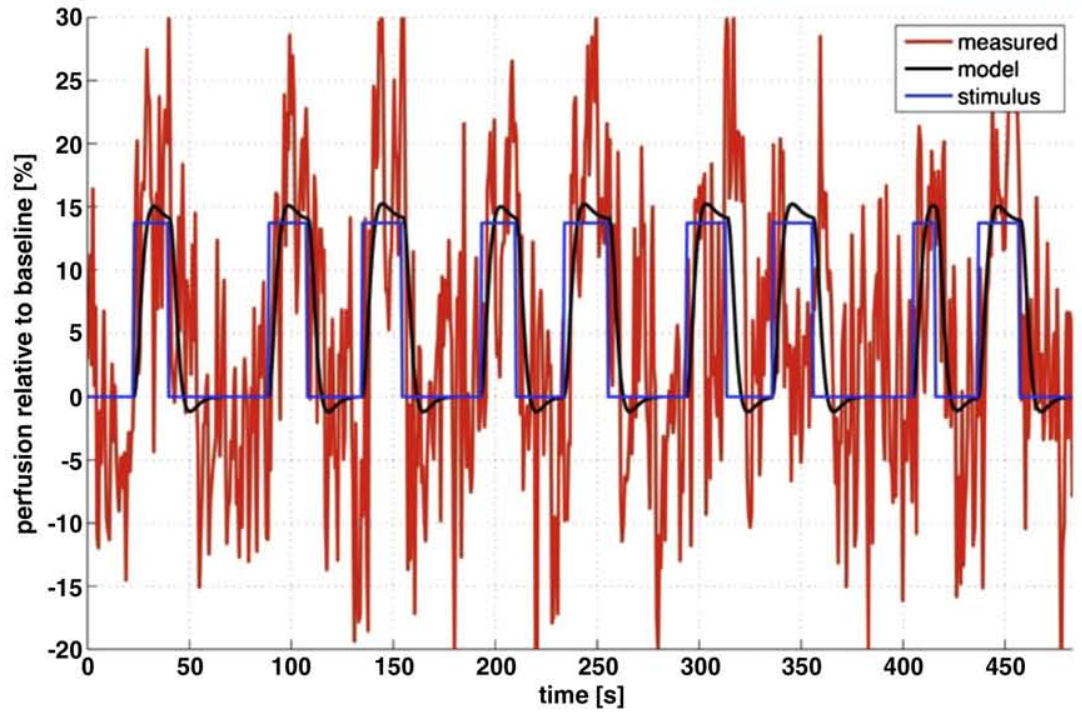

C

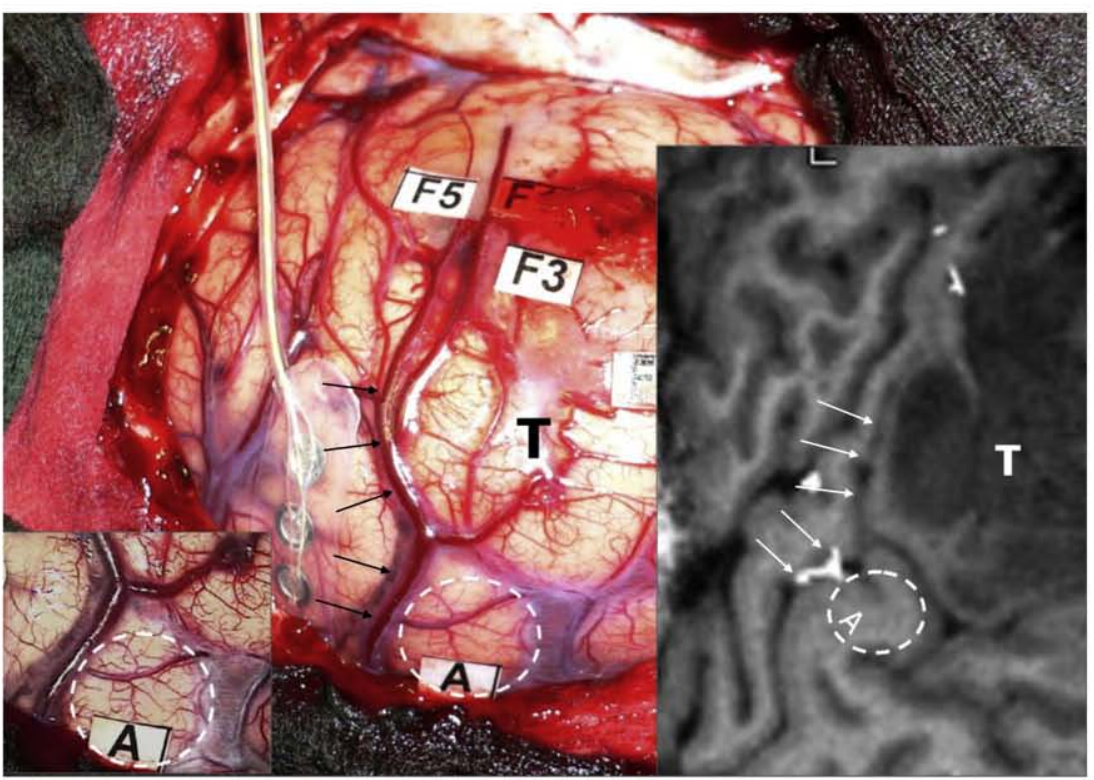


full area of the craniotomy and the corresponding MRI Mercator representation with fMRI activations (white) for a finger tapping task. The region correlates well with that detected by LDI as well as that determined by the cortical electro-stimulation. Although the response to the third task from the end is off (Fig. 3(b)) a robust fit was achieved demonstrating the strength of a statistical approach. The origin of this discrepancy is not clear but may lie in the natural variability in response, the difficulties in defining and fitting a baseline or even in the task performance (monitoring was not carried out precisely).

This case is representative of observations made during a series of surgical interventions and shows statistically significant activation areas which correlate with those observed in fMRI and electrostimulation, thereby demonstrating LDI to be a prospective method for surgical application and for brain research. A Laser emitting at $808 \mathrm{~nm}$ was chosen so that both oxy- and deoxy-hemoglobin could be probed equally, providing equal contributions to the image, a penetration depth of a few mm can be expected (Bevilacqua et al., 1999; Svaasand and Ellingsen, 1983). The cortex generally being of 1-4.5 $\mathrm{mm}$ in thickness (Fischl and Dale, 2000) we can expect to probe the fine branching vascular structure associated with the grey matter (Logothetis and Wandell, 2004). Laser Doppler imaging with a CMOS camera implies sampling scattered light from the population of moving red blood cells such that the image intensity is related to the number of red blood cells moving in a given volume and hence to perfusion, (Bonner and Nossal, 1981; Karamata et al., 2005), and the subtleties of diffuse scattering and multiple interactions are also taken into account. Thus LDI provides a relatively straight forward method for visualizing local variations in blood flow, the signal being distinguished from the background of light reflected from the brain surface. The alignment, GLM fitting and wavelet-based analysis allow the relevant data to be extracted and distinguished from confounding factors; breathing, pulse and theatre noise. The statistical testing ensures that the extracted map is significant and true. This has been found to be possible using an activation protocol with only very few repetitions. There needs however to be further work to deepen the understanding of the physiological origins of the LDI signal measured to ensure that an appropriate hemodynamic model is applied in consideration of the wavelength used.

The closest comparable intraoperative imaging technique is optical intrinsic signal imaging (OIS) which benefits from the faster frame rate of $\sim 15$ frames/s and a spatial resolution of $\sim 100 \mu \mathrm{m}$ allowing access to the evolution of the hemodynamic response in time and space; its contrast however derives from small changes in reflectance, less than $\sim 1 \%$ (Roe, 2007) and so achieves an attendant low SNR 7:1 requiring averaging over several trials (signal/standard deviation of signal at rest) (Pouratian et al., 2002b), furthermore the contrast is relatively complex to interpret having both hemodynamic and metabolic contributions coupled to neuronal activity. BOLD imaging of fMRI is a very powerful preoperative technique for brain-mapping but is extremely difficult to perform intraoperatively. Indocyanine green ICG may be used to assess cortical perfusion but only allows the in-flow of blood to be visualized before total image saturation, furthermore it is also invasive (Raabe et al., 2003).

LDI has proven to be relatively fast (frame rate of $1.48 \mathrm{~Hz}$ ), of relatively high spatial resolution $(\sim 290 \mu \mathrm{m})$ and good temporal response. In the current example the image sequence was acquired over $\sim 8 \mathrm{~min}$ although we have found that an acceptable fit may be achieved after only 3 epochs i.e. $\sim 3$ min imaging. The image postprocessing took 1-2 min. Further improvements in camera technology and data handling are expected to yield a higher spatiotemporal resolution as well as a more flexible instrument with which hemodynamics, small animal imaging and multiple wavelengths will be brought within reach. The LDI equipment may be easily integrated within standard surgical procedures and is not expected to disturb the course of surgery. It is non-invasive (class 1
Laser equipment), eliminates the risk of inducing seizure and, compared with electrostimulation, may considerably shorten the time required for mapping the exposed brain. This would allow multiple and more complex paradigms to be used in theatre and so superposable maps of different activations could be generated for injection into the surgical microscope ocular alleviating certain difficulties associated with brain-shift. Clearly fiber tract integrity will still need to be monitored as today with SEP, MEP or monopolar stimulation.

These are of course initial results from a prototype LDI and data analysis using fMRI style processing, there remains much work to be done to understand the contrast mechanisms and to ensure the correlation with the current surgical gold standard, cortical electrostimulation. Nevertheless, this represents a step towards the neurosurgeon's dream of visualizing the functional areas he strives to preserve in the surgical microscope, easily, on-line and with high spatiotemporal resolution, as well as facilitating the imaging of awake subjects necessary for research thanks to the strong and specific signal achieved.

\section{Acknowledgments}

The authors would like to thank R. Galuske of the University of Darmstadt for illuminating discussions. This work was supported in part by the Center for Biomedical Imaging (CIBM) of the GenevaLausanne Universities, the EPFL, and the foundations of Leenaards and Louis-Jeantet.

\section{References}

Berman, J.I., Berger, M.S., Mukherjee, P., Hernry, R.G., 2004. Diffusion-tensor imagingguided tracking of fibres of the pyramidal tract combined with intraoperative cortical stimulation mapping in patients with gliomas. J. Neurosurg. 101 (1), 66-72.

Bevilacqua, F., Piguet, D., Marquet, P., Gross, J.D., Tromberg, B.J., Depeursinge, C., 1999. In vivo local determination of tissue optical properties: applications to human brain. App. Opt. 38, 4939-4950.

Bonner, R., Nossal, R., 1981. Model for laser Doppler measurements of blood flow in tissue. Appl. Opt. 20, 2097-2107.

Bullmore, E., Brammer, M., Williams, S.C., Rabe-Hesketh, S., Janot, N., David, A., Mellers, J., Howard, R., Sham, P., 1996. Statistical methods of estimation and inference for functional MR image analysis. Magn. Reson. Med. 35 (2), 261-277.

Buxton, R.B., Frank, L.R., 1997. A model for the coupling between cerebral blood flow and oxygen metabolism during neural stimulation. J. Cereb. Blood Flow Metab. 17, 64-72.

Fischl, B., Dale, A.M., 2000. Measuring the thickness of the human cerebral cortex from magnetic resonance images. PNAS 97, 11050-11055.

Frackowiak, R.S.J., 2004. Human Brain Function, 1 ed. Academic Press.

Friston, K.J., Mechelli, A., Turner, R., Price, C.J., 2000. Nonlinear responses in fMRI: the balloon model, volterra kernels and other hemodynamics. NeuroImage 12, 466-477.

Haglund, M.M., Ojemann, G.A., Hochman, D.W., 1992. Optical imaging of epileptiform and functional activity in human cerebral cortex. Nature 358, 668-671.

Hillman, E.M.C., 2007. Optical brain imaging in vivo: techniques and applications from animal to man. J. Biomed. Opt. 12, 051402-051401 - 051428.

Iadecola, C., Nedergaard, M., 2007. Glial regulation of the cerebral microvasculature. Nat. Neurosci. 10, 1369-1376.

Karamata, B., Laubscher, M., Leutenegger, M., Bourquin, S., Lasser, T., Lambelet, P., 2005 Multiple scattering in optical coherence tomography. I. Investigation and modeling. JOSA A 22, 1369-1379.

Logothetis, N.K., Pfeuffer, J., 2004. On the nature of the BOLD fMRI contrast mechanism. Magn. Reson. Imaging 22, 1517-1531.

Logothetis, N.K., Wandell, B.A., 2004. Interpreting the BOLD signal. Annu. Rev. Physiol. $66,735-769$.

Obrig, H., Villringer, A., 2003. Beyond the visible-imaging the human brain with light J. Cereb. Blood Flow Metab. 23, 1-18.

Pouratian, N., Cannestra, F., Martin, N.A., Toga, A.W., 2002a. Intraoperative optical intrinsic signal imaging: a clinical tool for functional brain mapping. Neurosurgical Focus 13, 1-9.

Pouratian, N., Sheth, S.A., Martin, N.A., Toga, A.W., 2003. Shedding light on brain mapping: advances in human optical imaging. Trends Neurosci. 26, 277-282.

Pouratian, N., Sicotte, N., Rex, D., Martin, N.A., Becker, D., Cannestra, A.F., Toga, A.W. 2002b. Spatial/temporal correlation of BOLD and optical intrinsic signals in humans. Magn. Reson. Med. 47, 766-776.

Raabe, A., Beck, J., Gerlach, R., Zimmermann, M., Seifert, V., 2003. Near-infrared indocyanine green video angiography: a new method for intraoperative assessment of vascular flow. Neurosurgery 52, 132-139.

Roe, A.W., 2007. Long-term optical imaging of intrinsic signals in anesthetized and awake monkeys. Appl. Opt. 46, 1872-1880. 
Serov, A., Lasser, T., 2005. High-speed laser Doppler perfusion imaging using an integrating CMOS image sensor. Opt. Express 13, 6416-6428.

Svaasand, L.O., Ellingsen, R., 1983. Optical properties of human brain. Photochem. Photobiol. 38, 293-299.

Thévenaz, P., Ruttimann, U.E., Unser, M., 1998. A pyramid approach to subpixel registration based on intensity. IEEE Trans. Image Process. 7, 27-41.

Toga, A.W., Mazziotta, J.C., 2002. Brain Mapping, the Methods. Academic Press.
Van De Ville, D., Blu, T., Unser, M., 2004. Integrated wavelet processing and spatial statistical testing of fMRI data. NeuroImage 23, 1472-1485.

Vennemann, P., Lindken, R., Westerweel, J., 2007. In vivo whole-field blood velocity measurement techniques. Exp. Fluids 42, 495-511.

Wray, S., Cope, M., Delpy, D.T., 1988. Characterisation of the near infrared absorption spectra of cytochrome aa3 and haemoglobin for the non-invasive monitoring of cerebral oxygenation. Biochim. Biophys. Acta 933, 184-192. 